\title{
Ethnobotanical investigation of significant seasonal medicinal weeds of Toba Tek Singh District, Punjab, Pakistan
}

\author{
MUHAMMAD TUFAIL ${ }^{\circledR}$, KHALID HUSSAIN*®, KHALID NAWAZ, IQRA IQBAL, NOSHIA ARSHAD, \\ AMNA SHAHZADI, AYESHA JAVED, ARIFA NAZEER, ZOBIA BASHIR, MOHAMMAD QURBAN, \\ JAMAL ZEB, SYED SAQIB ALI
}

\author{
Department of Botany \\ University of Gujrat \\ Gujrat, Pakistan \\ *corresponding author: e-mail: khalid.hussain@uog.edu.pk
}

\section{Summary}

Introduction: Medicinal plants are found throughout the world but most are considered weeds. They are - directly or indirectly - the major source of medicines in pharmaceutical and herbal industries. Formulations used to prepare medicines or the method of use for these plants are mainly based on folk or traditional knowledge. This folk knowledge is not documented in many areas and needs to be explored.

Objectives: This study was aimed to enlist the seasonal weed species with traditional medicinal usage in Toba Tek Singh District, Punjab, Pakistan.

Methods: Field surveys were arranged in winter and summer 2019-2020 to enlist the important medicinal weeds and traditional knowledge of the local community. Data collected were as follows: local name of weed, medicinal use, method and part used.

Results: Numerous wild perennial, biennial and annual plants were identified, 30 of them were ethnomedicinally important in the local community. They were grouped in 16 families. It was found that whole weed is used in many prescriptions (37\%). Achyranthes aspera L. (Amaranthaceae) was the most common weed used in treating fevers, respiratory problems and asthma. Cichorium intybus L. (Asteraceae) was used in summer drinks to reduce thirst, improve digestion and liver function. Chenopodium album L. (Amaranthaceae) was used with $0.71 \mathrm{UV}$ and 0.147 RFC values. Medicago polymorpha L. (Fabaceae) was used to treat kidney, intestinal and bladder infections. Its UV was 0.65 and RFC was 0.121 . Tribulus terrestris L. (Zygophyllaceae) was used in impotency treatment, and in the removal of kidney stones and urinary tract infections treatment. It has $0.63 \mathrm{UV}$ and $0.21 \mathrm{RFC}$ values. This weed also showed the highest Fidelity Level (FL) (77\%), as compared to other weeds. 
Conclusion: It was concluded that there are many significant medicinal weeds in the Toba Tek Singh District, Punjab, Pakistan that are used in traditional medicines in treating various disorders. These plants also showed herbal or pharmacological importance that can be used to develop medicine at commercial scale.

Key words: weeds, ethnobotany, flora, survey

Słowa kluczowe: chwasty, etnobotanika, flora, badanie

\section{INTRODUCTION}

Ethnomedicinal surveys are of great significance in the development of drugs from indigenous medicinal plants [1]. Most of the medicinal plants are found as weeds, as compared to cultivated plants. That is why weeds are considered as major source of medicines. Weeds are important among local inhabitants in the management of many diseases [2]. Ethnomedicinal surveys are appropriate to document the useful medicinal information [3]. This documentation of traditional or folk knowledge of native weeds has contributed to the discovery of many vital drugs [4]. It is estimated that about $25 \%$ medicines are obtained directly or indirectly from plants [5]. Ethnomedicinal surveys helped the researchers find the significant plants in the specific areas that are used in folk medicines [2].

In the world, there are many useful plants which can have significant medicinal importance [1]. In Pakistan, there is a great diversity in medicinal plants which are a big source of medicines in local communities [6] The diversity concerns weather, terrestrial areas, conventional zones and flora [7]. Pakistan has predominantly huge, spotted and various diversity of medicinal plants. Shinwari [8] reported that about 6000 medicinal plants are available in Kashmir and Pakistan. Similarly, Khan et al. [9] described that these medicinal plants used in healthcare systems from ancient times. Folk medicines are important locally as well as are useful in pharmaceutical industry, worldwide [10]. Nearly 50,000 plants are used for medicinal purposes, worldwide [11]. Rural communities depend on indigenous plants in the treatment of many diseases [12]. Rehman et al. [2] described the flora of Gujrat district. Important weeds of this area have also been documented by Ali et al. [13].

There are only few reports describing the importance of weeds from various regions of Pakistan. The weeds of district Toba Tek Singh, Punjab, Pakistan have not been documented. That is why there was a need to document the useful weeds of the area with its medicinal significance. For this purpose, present study was conducted to document the weeds with its ethnomedicinal values in Toba Tek Singh District, Punjab, Pakistan.

\section{MATERIALS AND METHODS}

\section{Site of study}

This study was conducted in Toba Tek Singh District, Punjab province, Pakistan in summer and winter of 2019-2020 at a latitude of $30^{\circ} 33^{\prime}$ to $31^{\circ} 2^{\prime} \mathrm{N}$ and at longitude of $72^{\circ} 08^{\prime}$ to $72^{\circ} 48^{\prime} \mathrm{E}$ [1]. City is located at $162 \mathrm{~m}$ above sea level and consist of three tehsil that is Gojra, Toba Tek Singh and Kamalia (fig. 1).

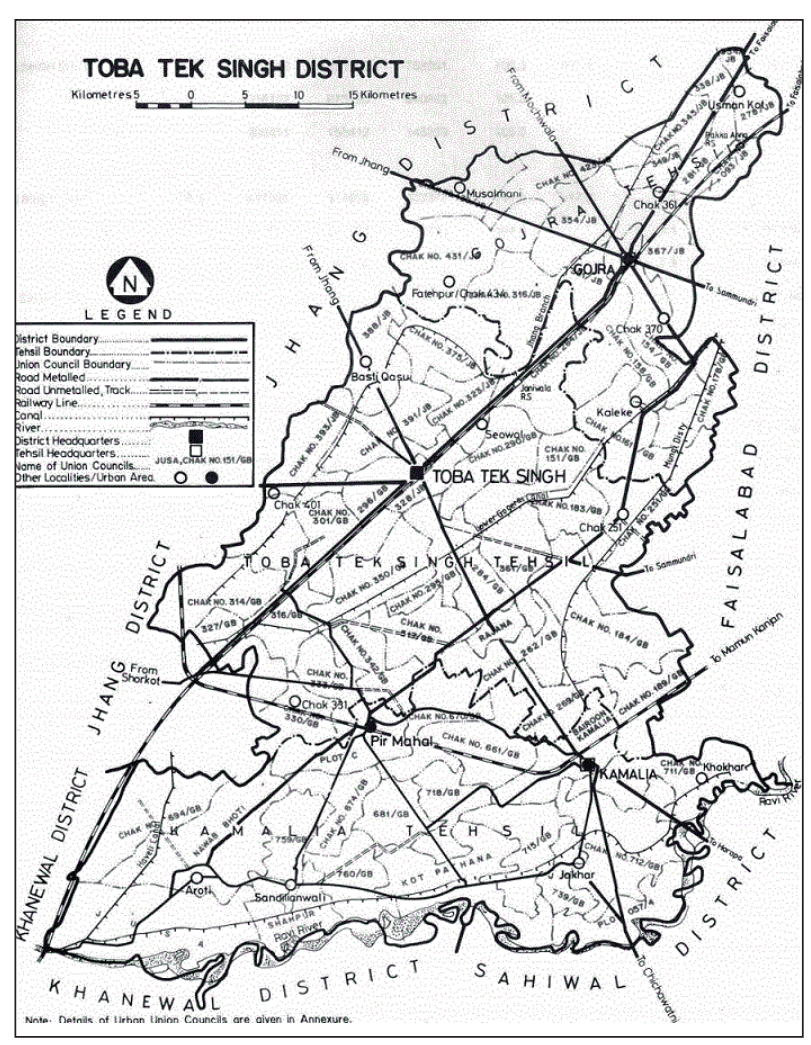

Figure 1.

Map of district Toba Tek Singh, Pakistan (study area). Map reference: https://www.politicpk.com/toba-tek-singh-district

There were a total of 25 visits performed and each visit lasted for 3-4 days. There were 18 study sites nearby rural areas where local community mainly uses weeds in treatment. Weed samples were collected with help of local guides. They were then collected 
and dried, placed in herbarium sheets and identified with the help of field guides with their local names.

\section{Collection of data}

Information about local names of the weeds and parts used for treating a specific disease was compiled through interviews and questionnaires. There were 75 respondents. The age of respondents ranged from 20 to 75 years, both male and female. They were local health providers (hakims). The questionnaire was designed to collect the desired ethnomedicinal information. Plants were identified with the help of reference plants preserved at herbarium, Department of Botany, University of Gujrat, Pakistan and the collected plant specimens have been deposited in this herbarium. They were further cross-verified through "Flora of Pakistan" [15].

\section{Data analysis}

Plant parts were divided into various categories, i.e. root, shoot, leaf and flower and data was analyzed using the following formulae:

\section{Use Value (UV)}

UV was determined to calculate relative importance of given species collected from the study area. It was calculated by the following formula:

$$
\mathrm{UV}=\Sigma \mathrm{U} / \mathrm{N}
$$

where $\mathrm{U}$ - number of citations per specific plant, $\mathrm{N}$ - number of local informants.

\section{Relative Frequency of Citation (RFC)}

RFC was calculated by the given formula:

$$
\mathrm{RFC}=\frac{\mathrm{FC}}{\mathrm{N}}
$$

where FC - the number of informants for the use of that species,

$\mathrm{N}$ - total number of informants in the survey.

\section{Informant Consensus Factor (ICF)}

Information of related homogeneity mixture of disease category collected from informants was calculated by the following formula:

$$
\mathrm{ICF}=\frac{\mathrm{Nur}-\mathrm{Nt}}{\mathrm{Nur}-1}
$$

where Nur -the number of use citations from informants for a particular plant-use category, $\mathrm{Nt}$ - the number of species or taxa utilized by all the informants for that specific plant use category.

ICF ranged from 0 to 1 , whereas ICF value 1 indicated the highest level of informant consent and 0 was the lowest value.

\section{Fidelity Level (FL)}

FL was used to determine the importance of the species as it relates to medicines.

$$
\operatorname{FL}(\%)=\frac{N p}{N} \times 100
$$

where $\mathrm{Np}$ - number of species that is present in the specific category,

$\mathrm{N}$ - accurate sum of consumption for a particular species.

Ethical approval: The conducted research is not related to either human or animal use.

\section{RESULTS}

In present study, 75 local inhabitants were interviewed and information was recorded through a questionnaire.

\section{Identification of plant families}

Weeds collected from Toba Tek Singh District belonged to 16 plant families. The area was found rich with trees, shrubs and herbs; however, 30 weeds were identified to have high ethnomedicinal use among local community. The families were: Asteraceae, Poaceae, Fabaceae, Polygonaceae, Amaranthaceae, Euphorbiaceae, Solanaceae, Brassicaceae, Convolvulaceae, Malvaceae, Oxalidaceae, Aizoaceae, Papaveraceae, Portulacaceae, Primulaceae and Zygophyllaceae (fig. 2). The families were ranked based on the number of weeds present in family. Fabaceae family was ranked at the top with 5 species, Asteraceae and Amaranthaceae with 4 species. There were 2 species of Poaceae and Euphorbiaceae, while all other families identified in the area had only one weed species. 


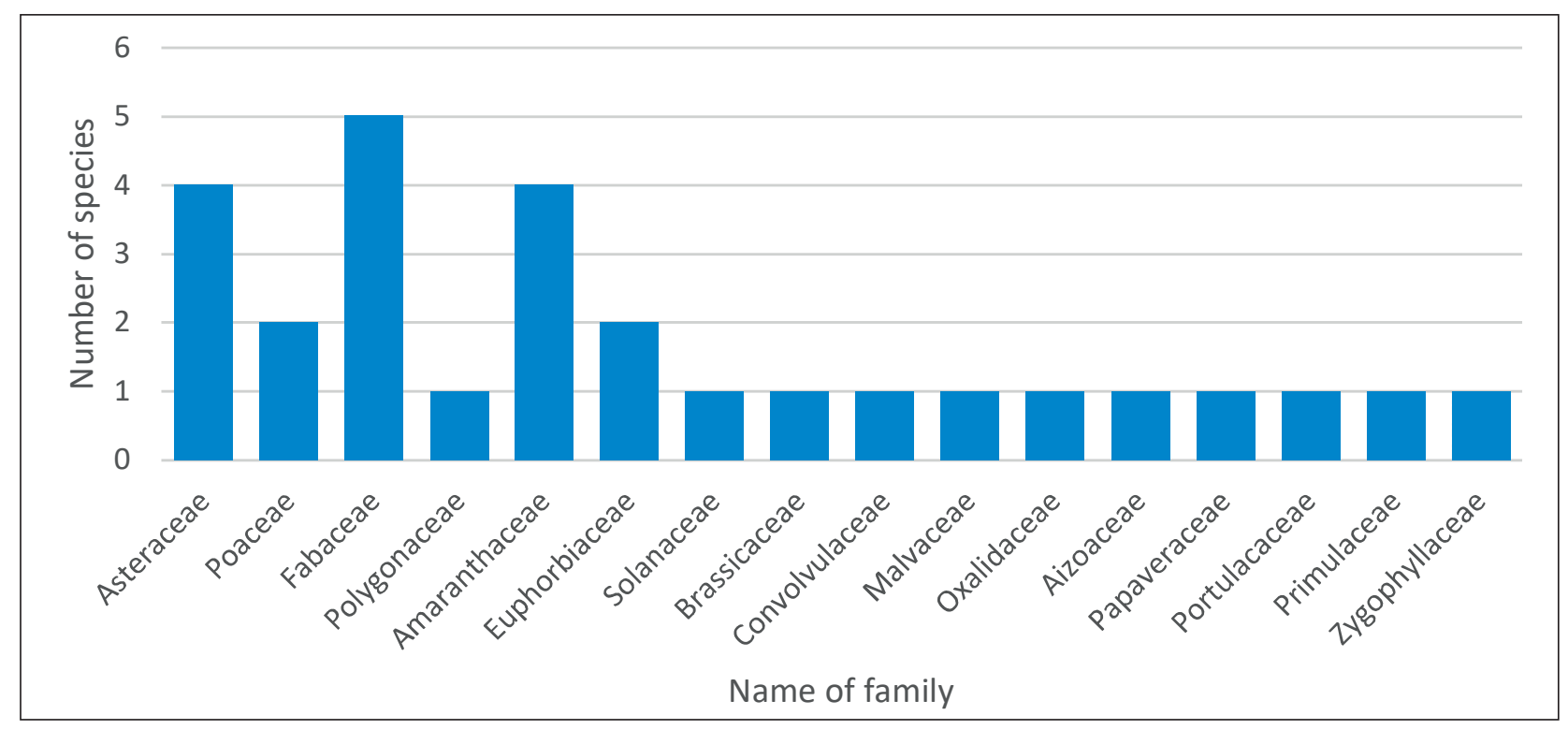

Figure 2.

Categories of families for collected weeds from district Toba Tek Singh, Pakistan

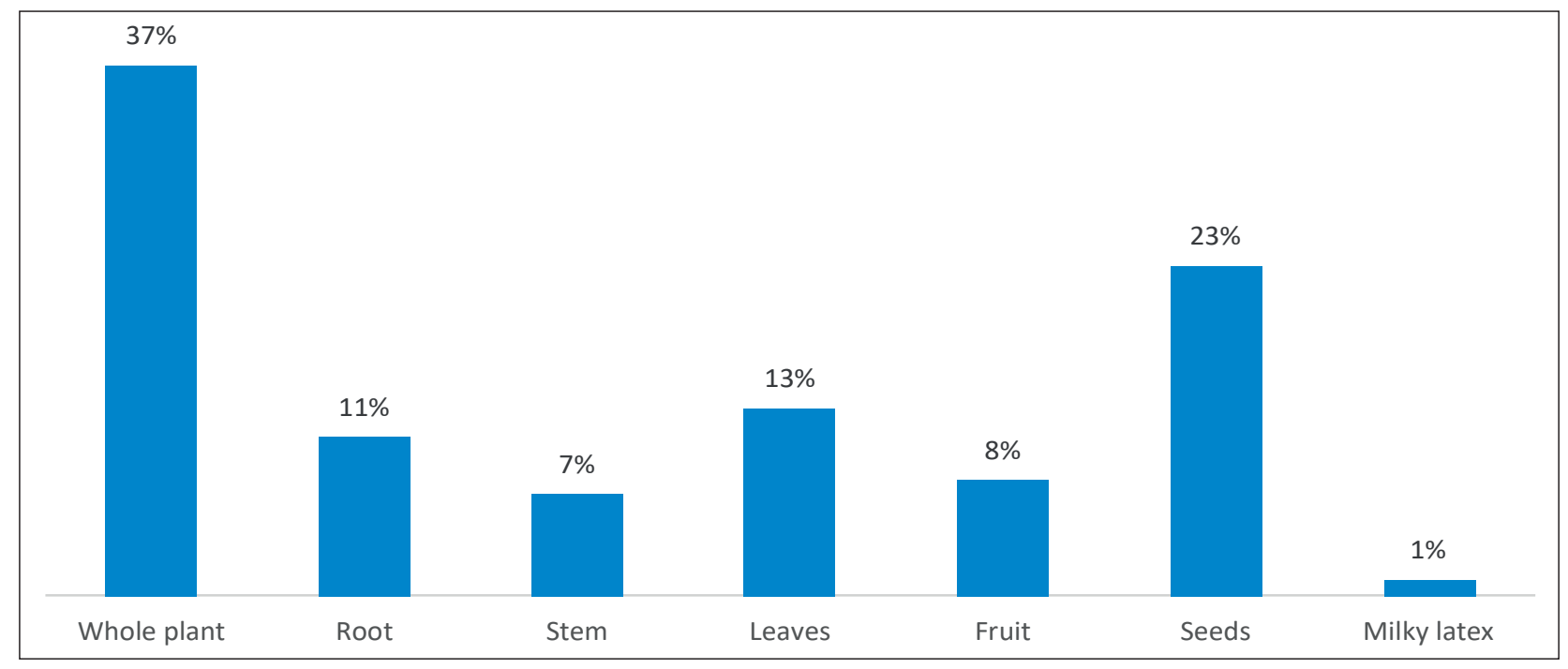

Figure 3.

Percentage of weed parts used for medicinal purpose collected from district Toba Tek Singh, Pakistan

\section{Parts used in ethnomedicine}

It was noted that different parts of weeds, i.e. roots, stem, leaves, latex, seeds and whole aerial parts were used to prepare different formulations. These parts were used both fresh and well dried in different medicines. Drugs from these plant parts were prepared in the form of infusion, extraction, paste powder, decoction and herbal tea. It was noted that various local medicine prescriptions used the following parts of plants: $37 \%$ whole plant, $23 \%$ seeds,
$13 \%$ leaves, $11 \%$ roots, $8 \%$ fruits, $7 \%$ stem and $1 \%$ latex (fig. 3).

\section{Ethnomedicinal uses}

During this study, information on botanical name, family, UV, RFC, uses, part used, prescription, dosage was collected (tab. 1). It was noted from local informants that all the plants growing in the area are of significance in the treatment of various diseases. 
Table 1.

Description of weeds with uses, method and prescription collected from district Toba Tek Singh, Pakistan

\begin{tabular}{|c|c|c|c|c|c|c|c|}
\hline $\begin{array}{l}\text { Botanical name of } \\
\text { weed }\end{array}$ & Name of family & Folk uses & $\begin{array}{l}\text { Prescription and part } \\
\text { used }\end{array}$ & $\begin{array}{l}\text { Method } \\
\text { of use }\end{array}$ & Doses & $\begin{array}{l}\text { Use value } \\
\text { (UV) }\end{array}$ & $\begin{array}{c}\text { Relative } \\
\text { Frequency } \\
\text { of Citation } \\
\text { (RFC) }\end{array}$ \\
\hline $\begin{array}{l}\text { Achyranthes } \\
\text { aspera L. }\end{array}$ & Amaranthaceae & $\begin{array}{l}\text { All type of fevers, } \\
\text { respiratory problems, } \\
\text { cough, asthma }\end{array}$ & $\begin{array}{l}\text { Fresh or dry leaves } \\
\text { are mixed with honey } \\
\text { and black pepper }\end{array}$ & $\begin{array}{l}\text { Internal } \\
\text { use }\end{array}$ & $\begin{array}{l}3 \text { times } \\
\text { per day }\end{array}$ & 0.82 & 0.084 \\
\hline $\begin{array}{l}\text { Amaranthus } \\
\text { viridis L. }\end{array}$ & Amaranthaceae & $\begin{array}{l}\text { Malaria, insect bite, } \\
\text { skin rash }\end{array}$ & $\begin{array}{l}\text { Seeds are mixed with } \\
\text { rice or honey to make } \\
\text { paste }\end{array}$ & $\begin{array}{l}\text { Internal } \\
\text { use }\end{array}$ & $\begin{array}{l}2 \text { times } \\
\text { per day }\end{array}$ & 0.34 & 0.046 \\
\hline $\begin{array}{l}\text { Anagallis } \\
\text { arvensis } \mathrm{L} .\end{array}$ & Primulaceae & $\begin{array}{l}\text { Wound healings, skin } \\
\text { infections }\end{array}$ & $\begin{array}{l}\text { Fresh leaves to make } \\
\text { poultice }\end{array}$ & $\begin{array}{l}\text { External } \\
\text { use }\end{array}$ & $\begin{array}{l}2 \text { times } \\
\text { per day }\end{array}$ & 0.33 & 0.089 \\
\hline Avena sativa $\mathrm{L}$. & Poaceae & $\begin{array}{l}\text { Stomach and digestive } \\
\text { problems, diabetes }\end{array}$ & $\begin{array}{l}\text { Seeds are grinded } \\
\text { with sugar to make } \\
\text { tablets }\end{array}$ & $\begin{array}{l}\text { Internal } \\
\text { use }\end{array}$ & $\begin{array}{l}1 \text { time } \\
\text { per day }\end{array}$ & 0.52 & 0.085 \\
\hline $\begin{array}{l}\text { Chenopodium } \\
\text { album } \mathrm{L} \text {. }\end{array}$ & Amaranthaceae & $\begin{array}{l}\text { Stomach/gastric and } \\
\text { urinary disorders }\end{array}$ & $\begin{array}{l}\text { Leaves are mixed } \\
\text { with water for kidney } \\
\text { disorder. Herbal tea } \\
\text { is useful }\end{array}$ & $\begin{array}{l}\text { Internal } \\
\text { use }\end{array}$ & $\begin{array}{l}2 \text { times } \\
\text { per day }\end{array}$ & 0.71 & 0.147 \\
\hline $\begin{array}{l}\text { Chenopodium } \\
\text { murale } \\
\text { (L.) S. Fuentes, } \\
\text { Uotila \& Borsch }\end{array}$ & Amaranthaceae & $\begin{array}{l}\text { Dry cough, respira- } \\
\text { tory or pulmonary } \\
\text { obstruction }\end{array}$ & $\begin{array}{l}\text { Whole plant part are } \\
\text { recommended to rub } \\
\text { at chest }\end{array}$ & $\begin{array}{l}\text { External } \\
\text { use }\end{array}$ & $\begin{array}{l}2 \text { times } \\
\text { per day }\end{array}$ & 0.59 & 0.051 \\
\hline $\begin{array}{l}\text { Cichorium } \\
\text { intybus L. }\end{array}$ & Asteraceae & $\begin{array}{l}\text { Shows cooling effects, } \\
\text { reduce thirst, improve } \\
\text { digestion and liver } \\
\text { functioning }\end{array}$ & $\begin{array}{l}\text { Fresh or dry leaves } \\
\text { are used to make } \\
\text { herbal tea to improve } \\
\text { digestion. Used as } \\
\text { summer drink }\end{array}$ & $\begin{array}{l}\text { Internal } \\
\text { use }\end{array}$ & $\begin{array}{l}2 \text { times } \\
\text { per day }\end{array}$ & 0.78 & 0.137 \\
\hline $\begin{array}{l}\text { Cirsium arvense } \\
\text { (L.) Scop. }\end{array}$ & Asteraceae & $\begin{array}{l}\text { Improves digestion } \\
\text { and used for child } \\
\text { worms }\end{array}$ & $\begin{array}{l}\text { Roots are used in } \\
\text { decoction }\end{array}$ & $\begin{array}{l}\text { Internal } \\
\text { use }\end{array}$ & $\begin{array}{l}3 \text { times } \\
\text { per day }\end{array}$ & 0.48 & 0.18 \\
\hline $\begin{array}{l}\text { Convolvulus } \\
\text { arvensis L. }\end{array}$ & Convolvulaceae & $\begin{array}{l}\text { Useful in urinary tract } \\
\text { infections and stom- } \\
\text { ach ulcers }\end{array}$ & $\begin{array}{l}\text { Root juice is recom- } \\
\text { mended }\end{array}$ & $\begin{array}{l}\text { Internal } \\
\text { use }\end{array}$ & $\begin{array}{l}2 \text { times } \\
\text { per day }\end{array}$ & 0.45 & 0.08 \\
\hline $\begin{array}{l}\text { Cyperus } \\
\text { rotundus } \mathrm{L} .\end{array}$ & Cyperaceae & $\begin{array}{l}\text { Anti-diabetic, anti-in- } \\
\text { flammatory and anti- } \\
\text { malarial properties }\end{array}$ & $\begin{array}{l}\text { Grind its rhizome to } \\
\text { make powder }\end{array}$ & $\begin{array}{l}\text { Internal } \\
\text { use }\end{array}$ & $\begin{array}{l}1 \text { time } \\
\text { per day }\end{array}$ & 0.26 & 0.07 \\
\hline $\begin{array}{l}\text { Digitaria bicornis } \\
\text { (Lam.) Roem. \& } \\
\text { Schult. }\end{array}$ & Poaceae & $\begin{array}{l}\text { Used to kill microbes } \\
\text { of intestine }\end{array}$ & $\begin{array}{l}\text { Leaves and seeds } \\
\text { are grinded to make } \\
\text { poultice }\end{array}$ & $\begin{array}{l}\text { External } \\
\text { use }\end{array}$ & $\begin{array}{l}1 \text { time } \\
\text { per day }\end{array}$ & 0.34 & 0.25 \\
\hline Eclipta prostrata L. & Asteraceae & $\begin{array}{l}\text { Anti-bacterial and } \\
\text { anti-oxidant. Useful } \\
\text { in fevers }\end{array}$ & $\begin{array}{l}\text { Whole plant is mixed } \\
\text { with olive oil }\end{array}$ & $\begin{array}{l}\text { Internal } \\
\text { use }\end{array}$ & $\begin{array}{l}1 \text { time } \\
\text { per day }\end{array}$ & 0.31 & 0.15 \\
\hline $\begin{array}{l}\text { Euphorbia } \\
\text { helioscopia L. }\end{array}$ & Euphorbiaceae & $\begin{array}{l}\text { Used in constipation, } \\
\text { athlete's foot and in- } \\
\text { testinal problems. }\end{array}$ & $\begin{array}{l}\text { Leaves are used as } \\
\text { herbal tea }\end{array}$ & $\begin{array}{l}\text { Internal } \\
\text { use }\end{array}$ & $\begin{array}{l}3 \text { times } \\
\text { per day }\end{array}$ & 0.54 & 0.13 \\
\hline $\begin{array}{l}\text { Euphorbia prostrata } \\
\text { Aiton }\end{array}$ & Euphorbiaceae & $\begin{array}{l}\text { Recommended in } \\
\text { jaundice, fever and } \\
\text { kill intestinal worms. }\end{array}$ & $\begin{array}{l}\text { Aerial parts are used } \\
\text { to make powder or } \\
\text { tablets }\end{array}$ & $\begin{array}{l}\text { Internal } \\
\text { use }\end{array}$ & $\begin{array}{l}2 \text { times } \\
\text { per day }\end{array}$ & 0.51 & 0.16 \\
\hline $\begin{array}{l}\text { Fumaria indica } \\
\text { (Hausskn.) Pugsley }\end{array}$ & Papaveraceae & $\begin{array}{l}\text { Used in fever, cures } \\
\text { liver disorder and } \\
\text { diarrhea }\end{array}$ & $\begin{array}{l}\text { Leaves are boiled in } \\
\text { water to make decoc- } \\
\text { tion }\end{array}$ & $\begin{array}{l}\text { Internal } \\
\text { use }\end{array}$ & $\begin{array}{l}2 \text { times } \\
\text { per day }\end{array}$ & 0.62 & 0.041 \\
\hline Lathyrus aphaca L. & Fabaceae & $\begin{array}{l}\text { Useful to skin infec- } \\
\text { tion and used in in- } \\
\text { sect bites }\end{array}$ & $\begin{array}{l}\text { Seeds are grinded and } \\
\text { mixed with water }\end{array}$ & $\begin{array}{l}\text { Internal } \\
\text { use }\end{array}$ & $\begin{array}{l}1 \text { time } \\
\text { per day }\end{array}$ & 0.24 & 0.05 \\
\hline
\end{tabular}


Table 1. (continued)

\begin{tabular}{|c|c|c|c|c|c|c|c|}
\hline $\begin{array}{l}\text { Botanical name of } \\
\text { weed }\end{array}$ & Name of family & Folk uses & $\begin{array}{l}\text { Prescription and part } \\
\text { used }\end{array}$ & $\begin{array}{l}\text { Method } \\
\text { of use }\end{array}$ & Doses & $\begin{array}{l}\text { Use value } \\
\text { (UV) }\end{array}$ & $\begin{array}{c}\text { Relative } \\
\text { Frequency } \\
\text { of Citation } \\
\text { (RFC) }\end{array}$ \\
\hline $\begin{array}{l}\text { Lepidium } \\
\text { didymium L. }\end{array}$ & Brassicaceae & $\begin{array}{l}\text { Useful for the treat- } \\
\text { ment of gastrointesti- } \\
\text { nal ulcers }\end{array}$ & $\begin{array}{l}\text { Poultice of the leaves } \\
\text { is applied }\end{array}$ & $\begin{array}{l}\text { External } \\
\text { use }\end{array}$ & $\begin{array}{l}2 \text { times } \\
\text { per day }\end{array}$ & 0.55 & 0.14 \\
\hline Malva parviflora L. & Malvaceae & $\begin{array}{l}\text { Antimicrobial, anti- } \\
\text { bacterial and anti-in- } \\
\text { flammatory properties }\end{array}$ & $\begin{array}{l}\text { Leaves are boiled } \\
\text { in } 3-4 \text { cup water and } \\
\text { then are mixed in } \\
\text { cow milk }\end{array}$ & $\begin{array}{l}\text { Internal } \\
\text { use }\end{array}$ & $\begin{array}{l}\text { Daily } \\
\text { in the } \\
\text { evening }\end{array}$ & 0.22 & 0.14 \\
\hline $\begin{array}{l}\text { Medicago } \\
\text { polymorpha L. }\end{array}$ & Fabaceae & $\begin{array}{l}\text { Improves kidney, } \\
\text { intestinal and bladder } \\
\text { infections }\end{array}$ & $\begin{array}{l}\text { Powder of seeds are } \\
\text { mixed with water to } \\
\text { make a mush }\end{array}$ & $\begin{array}{l}\text { Internal } \\
\text { use }\end{array}$ & $\begin{array}{l}2 \text { times } \\
\text { per day }\end{array}$ & 0.65 & 0.121 \\
\hline Medicago sativa L. & Fabaceae & $\begin{array}{l}\text { Enhance metabolism } \\
\text { and increase milk pro- } \\
\text { duction in livestock }\end{array}$ & $\begin{array}{l}\text { Whole plant is boiled } \\
\text { with oat, alfalfa to } \\
\text { make a syrup }\end{array}$ & $\begin{array}{l}\text { Internal } \\
\text { use }\end{array}$ & $\begin{array}{l}2 \text { times } \\
\text { per day }\end{array}$ & 0.51 & 0.25 \\
\hline $\begin{array}{l}\text { Melilotus indicus } \\
\text { (L.) All. }\end{array}$ & Fabaceae & $\begin{array}{l}\text { Useful in diarrhea and } \\
\text { bowl complaints, in- } \\
\text { testinal problems }\end{array}$ & $\begin{array}{l}\text { Seeds or whole plant } \\
\text { is used to make tab- } \\
\text { lets or dry powder }\end{array}$ & $\begin{array}{l}\text { Internal } \\
\text { use }\end{array}$ & $\begin{array}{l}1 \text { time } \\
\text { per day }\end{array}$ & 0.54 & 0.35 \\
\hline $\begin{array}{l}\text { Oxalis } \\
\text { corniculata L. }\end{array}$ & Oxalidaceae & $\begin{array}{l}\text { Cures redness of eyes } \\
\text { and scurvy, and anti- } \\
\text { microbial activity }\end{array}$ & $\begin{array}{l}\text { Dry leaves are used } \\
\text { to make powder }\end{array}$ & $\begin{array}{l}\text { Internal } \\
\text { use }\end{array}$ & $\begin{array}{l}2 \text { times } \\
\text { per day }\end{array}$ & 0.54 & 0.16 \\
\hline $\begin{array}{l}\text { Portulaca } \\
\text { oleracea L. }\end{array}$ & Portulacaceae & $\begin{array}{l}\text { Recommended in } \\
\text { fever, ulcer and abnor- } \\
\text { mal uterine bleeding }\end{array}$ & $\begin{array}{l}\text { Whole plant is used } \\
\text { to make poultice }\end{array}$ & $\begin{array}{l}\text { Internal } \\
\text { use }\end{array}$ & $\begin{array}{l}1 \text { time } \\
\text { per day }\end{array}$ & 0.62 & 0.24 \\
\hline Rumex dentatus L. & Polygonaceae & $\begin{array}{l}\text { It is a good appetizer, } \\
\text { cure constipation and } \\
\text { diarrhea }\end{array}$ & $\begin{array}{l}\text { Leave juice is used. } \\
\text { Leaves are also } \\
\text { cooked vegetable }\end{array}$ & $\begin{array}{l}\text { External } \\
\text { use }\end{array}$ & $\begin{array}{l}2 \text { times } \\
\text { per day }\end{array}$ & 0.55 & 0.25 \\
\hline Solanum nigrum L. & Solanaceae & $\begin{array}{l}\text { Cures respiratory, } \\
\text { hepatic and stomach } \\
\text { disorders. Cure eye } \\
\text { piles and diabetes }\end{array}$ & $\begin{array}{l}\text { Leaves are mixed with } \\
\text { honey }\end{array}$ & $\begin{array}{l}\text { Internal } \\
\text { use }\end{array}$ & $\begin{array}{l}2 \text { times } \\
\text { per day }\end{array}$ & 0.43 & 0.16 \\
\hline $\begin{array}{l}\text { Sonchus asper (L.) } \\
\text { Hill }\end{array}$ & Asteraceae & $\begin{array}{l}\text { Antimicrobial activity, } \\
\text { paste is useful to cure } \\
\text { wounds }\end{array}$ & $\begin{array}{l}\text { Seeds are soaked in } \\
1 \text { cup water for one } \\
\text { night }\end{array}$ & $\begin{array}{l}\text { External } \\
\text { use }\end{array}$ & $\begin{array}{l}1 \text { time } \\
\text { per day }\end{array}$ & 0.38 & 0.08 \\
\hline $\begin{array}{l}\text { Trianthema } \\
\text { portulacastrum } \\
\text { L. }\end{array}$ & Aizoaceae & $\begin{array}{l}\text { Useful for the treat- } \\
\text { ment of constipation, } \\
\text { asthma }\end{array}$ & $\begin{array}{l}\text { Plant juice is extract- } \\
\text { ed and mixed with } \\
\text { sugar }\end{array}$ & $\begin{array}{l}\text { Internal } \\
\text { use }\end{array}$ & $\begin{array}{l}3 \text { times } \\
\text { per day }\end{array}$ & 0.541 & 0.14 \\
\hline Tribulus terrestris L. & Zygophyllaceae & $\begin{array}{l}\text { Used in impotency, } \\
\text { men sexual power. Re- } \\
\text { moves kidney stones } \\
\text { and urinary tract in- } \\
\text { fections }\end{array}$ & $\begin{array}{l}\text { Extract of leaves is } \\
\text { used. Seeds are also } \\
\text { sued to make powder }\end{array}$ & $\begin{array}{l}\text { Internal } \\
\text { use }\end{array}$ & $\begin{array}{l}1 \text { time } \\
\text { per day }\end{array}$ & 0.63 & 0.21 \\
\hline Vicia sativa $\mathrm{L}$. & Fabaceae & $\begin{array}{l}\text { Plant has anti-inflam- } \\
\text { matory and antioxi- } \\
\text { dant properties }\end{array}$ & $\begin{array}{l}\text { Poultice is recom- } \\
\text { mended against skin } \\
\text { infection }\end{array}$ & $\begin{array}{l}\text { External } \\
\text { use }\end{array}$ & $\begin{array}{l}1 \text { time } \\
\text { per day }\end{array}$ & 0.41 & 0.13 \\
\hline
\end{tabular}

From various ethnomedicinal data analysis it was found that there were 30 weeds in the study area. Most common were Achyranthes aspera, Amaranthus viridis, Anagallis arvensis, Avena sativa, Chenopodium album, Chenopodium murale, Cichorium intybus, Cirsium arvense, Convolvulus arvensis, Cyperus rotundus and Digitaria bicornis.
It was found that Achyranthes aspera (Amaranthaceae) was the most common weed used to treat fevers, respiratory tract problems and asthma with $0.82 \mathrm{UV}$ and $0.084 \mathrm{RFC}$ values. Cichorium intybus (Asteraceae) showed 0.78 UV and 0.137 RFC values and was used to in summer drinks to reduce thirst, improve digestion and liver functions. 
Chenopodium album (Amaranthaceae) was used with $0.71 \mathrm{UV}$ and 0.147 RFC values. Medicago polymorpha (Fabaceae) was used to improve kidney, intestinal and bladder infections. Its UV was 0.65 and RFC was 0.121 . Tribulus terrestris (Zygophyllaceae) was used in impotency treatment. It also helps to remove kidney stones and treat urinary tract infections. It has $0.63 \mathrm{UV}$ and $0.21 \mathrm{RFC}$ values. This weed also showed the highest FL value (77\%), as compared to other weeds (tab. 1).

\section{Pharmacological data analysis}

Informant Consensus Factor (ICF) by disease category and Fidelity Level (FL) showed values ranging from 77 to 51 for most reported medicinal weeds (tab. 2, 3). According to respondents and calculated ICF values, these weeds were recommended in respiratory tract diseases, gastric problems, kidney and liver disorders, diarrhoea, constipation, fever, cold, cough, asthma, flue, intestinal problems, arthritis, malaria, cancer, allergies, male infertility, sexual

Table 2.

Informant Consensus Factor (ICF) by disease category in district Toba Tek Singh, Pakistan

\begin{tabular}{|c|c|c|c|c|c|}
\hline Disease Category & $\begin{array}{l}\text { No. of Use } \\
\text { Report } \\
\text { (Nur) }\end{array}$ & $\begin{array}{c}\text { No. of } \\
\text { Species } \\
\text { Used (Nt) }\end{array}$ & Nur-Nt & Nur-1 & ICF \\
\hline Asthma, flue, cold, cough, respiratory infection and fever & 61 & 35 & 26 & 64 & 0.43 \\
\hline Bone pain, joint swellings, jaundice & 32 & 17 & 15 & 21 & 0.24 \\
\hline Liver disorders & 31 & 13 & 18 & 27 & 0.57 \\
\hline Headache, hypertension & 15 & 9 & 6 & 19 & 0.63 \\
\hline Insect bite, body pain, epilepsy, convulsion, infections & 21 & 5 & 16 & 16 & 0.69 \\
\hline Jaundice, malaria, bronchitis, vomiting, intestinal problems, arthritis & 22 & 17 & 5 & 24 & 0.43 \\
\hline Kidney and liver disorders & 33 & 11 & 22 & 28 & 0.58 \\
\hline Loose motion, constipation & 45 & 22 & 23 & 41 & 0.53 \\
\hline Malaria, cancer, peralysis, tumor, allergies & 19 & 14 & 5 & 23 & 0.42 \\
\hline Piles, snake bite, dropsy', cholera, toothache, rheumatism & 23 & 13 & 10 & 22 & 0.54 \\
\hline Respiratory diseases, gastric problems, hepatitis & 21 & 14 & 7 & 20 & 0.57 \\
\hline Stomach problems, gastric problems, intestinal problem, digestive problems & 32 & 8 & 24 & 32 & 0.71 \\
\hline Wound healing, skin diseases or inflammation & 62 & 35 & 27 & 61 & 0.48 \\
\hline
\end{tabular}

Nur - number of citations from informants for a particular plant-use category; $\mathrm{Nt}$ - number of species

Table 3.

Fidelity Level (FL) value for most reported medicinal weeds in district Toba Tek Singh, Pakistan

\begin{tabular}{lllc}
\multicolumn{1}{c}{ Botanical name of weed } & Local name & \multicolumn{1}{c}{ Major ailment } & $\begin{array}{c}\text { Fidelity } \\
\text { level (FL) \% }\end{array}$ \\
\hline Achyranthes aspera L. & Putkanda & All type of fevers, respiratory problems, cough, asthma & 74 \\
\hline Amaranthus viridis L. & Ghunar & Malaria, insect bite, skin rash & 59 \\
\hline Anagallis arvensis L. & Bili booti & Wound healings, skin infections & 54 \\
\hline Chenopodium album L. & Bathu & Stomach/gastric and urinary disorders & 75 \\
\hline Cichorium intybus L. & Kasani & Reduces thirst, improve digestion and liver functioning & 65 \\
\hline Eclipta prostrata L. & Bhangra & Anti-bacterial and anti-oxidant. Useful in fevers & 51 \\
\hline Fumaria indica (Hausskn.) Pugsley & Shahtra & Used in fever, cures liver disorder and diarrhea & 72 \\
\hline Rumex dentatus L. & Jangli palak & It is a good appetizer, cure constipation and diarrhea & 61 \\
\hline Solanum nigrum L. & Kainch mainch & $\begin{array}{l}\text { Cures respiratory, hepatic and stomach disorders. Cure eye } \\
\text { piles and diabetes }\end{array}$ & 69 \\
\hline Trianthema portulacastrum L. & Itsit & Useful for the treatment of constipation, asthma & 71 \\
\hline Tribulus terrestris L. & Bhukhra & $\begin{array}{l}\text { Used in impotency, men sexual power. Removes kidney } \\
\text { stones and urinary tract infections }\end{array}$ & 77 \\
\hline
\end{tabular}


disorders, piles, snake bite, stomach problems, gastric problems, intestinal problem, digestive problems, insect bite, body pain, epilepsy, convulsion, infections, nervous disorders, swellings and pneumonia (tab. 2).

The FL value of most reported weeds have been given in table 3. Tribulus terrestris showed the highest FL value $(77 \%)$ when used in impotency treatment. It is helpful in removal of kidney stones and in treatment of urinary tract infections. Similarly, a $75 \%$ FL value was noted for Chenopodium album recommended for gastric problems.

\section{DISCUSSION}

Present study revealed that there are many seasonal weeds, e.g. Achyranthes aspera, Amaranthus viridis, Anagallis arvensis, Avena sativa, Chenopodium album, Chenopodium murale, Cichorium intybus, Cirsium arvense, Convolvulus arvensis, Cyperus rotundus and Digitaria bicornis that have not been documented earlier in Toba Tek Singh District, Pakistan. Similarly, Rehman et al. [2] reported 32 families of weeds and showed the significance of seasonal weeds growing in Gujrat District, Pakistan against many diseases by local inhabitants. Muhammad et al. [14] described the weeds of wheat crop that had great significance in the treatment of various diseases in Tehsil Gojra, Pakistan. Pakistan has different seasons and a variety of soils, which is why the country is rich with medicinal plants.

It has been described that in most areas local communities rely on these plants to treat many diseases. The use of plants is based upon folk knowledge. As it was noted, the area had important wild flora belonging to various families. Similar results have been found in previous studies. Saurabh et al. [16] described that Achyranthes aspera L. is useful in treating asthma, piles, dropsy, snake bite, rheumatism and skin diseases. Amaranthus viridis (Amaranthaceae) was useful in the treatment of malaria, useful for prolapse of uterus, urinary tract infections, snake bite [17]. During this study it was noted that Avena sativa (Poaceae) is considered to have antioxidant and wound healing properties which is in accordance with the earlier findings of Akkol et al. [18]. Calotropis procera Aiton. has anti-diarrhoeal and anti-inflammatory activity [19]. Mishra et al. [20] described that the paste of Fumaria parviflora var. indica (Papaveraceae) was useful for treating joint swellings. All the plants documented in this area showed many values for different pharmacological attributes which have been also observed in the past. Rehman et al. [2] observed that the most common medicinal plant used by local people was Cichorium intybus which had a $100 \%$ FL value when used as a liver tonic and blood purifier. Highest $0.76 \mathrm{ICF}$ values were noted against stomach, gastric, intestinal and digestive problems. Achyranthes aspera showed the highest FL value (93\%) when used in gastrointestinal disorders and menstrual pain. Padmavathi et al. [21] claimed that the high FL value showed the use of a particular weed to treat a specific disease. Rehman et al. [2] reported the significance of weeds in traditional medicines. They described 33 families with important weeds of the area that are used to treat various diseases including skin infection, respiratory and asthma problems, kidney, liver and heart diseases. Shinwari and Khan [22] recorded fifty species of herbs belonging to 27 families used medicinally by people native to Margalla Hills of National Park in Islamabad. From the total of 50 species, only 10 species were being sold in local market. Ahmad et al. [23] studied 6 important plant species of medicinal importance. Achyranthes aspera was used as purgative, laxative, diuretic, antiviral and styptic agent.

There are many reports describing the importance of weeds in other regions of Pakistan. Hussain et al. [7] reported 40 plant species from 39 genera and 32 families having medicinal properties in Haripur region of Pakistan. They described that Acacia arabica var. nilotica (L.) Benth. was used as astringent, tonic, antiseptic and purgative agent. Khan et al. [24] reported that Calotropis procera (Apocynaceae) were useful in cough, rheumatism and skin infection. Qureshi et al. [25] reported 63 herbs belonging to 50 genera and 29 families having medicinal importance.

It has been confirmed that weeds have significance in folk medicines. Local community depends on these weeds based upon traditional knowledge.

\section{CONCLUSIONS}

It was concluded that Toba Tek Singh District, Punjab, Pakistan has many weeds that are used in folk medicines to treat many diseases. There is a need to establish a link among local community, researchers and herbalist to develop medicine with mutual knowledge sharing about these weeds. 


\section{ACKNOWLEDGEMENT}

All authors would like to thank the local people of the area and staff of the Department of Botany, University of Gujrat, Pakistan for their support during this study.

Conflict of interest: Authors declare no conflict of interest.

\section{REFERENCES}

1. Tufail M, Hussain K, Nawaz K, Bhatti KH, Yasin G, Ali SS. Ethnobotanical survey of important wild medicinal plants of tehsil Gojra, District Toba Tek Singh, Punjab, Pakistan. Ethnobot Res Appl 2020; 1-14. doi: https://dx.doi.org/10.32859/ era.20.23.1-14

2. Rehman A, Hussain, K, Nawaz K, Arshad N, Iqbal I, Ali SS et al. Indigenous knowledge and medicinal significance of seasonal weeds of district Gujrat, Punjab, Pakistan. Ethnobot Res Appl 2020; 20:1-19. doi: http://dx.doi.org/10.32859/ era.20.12.1-19

3. Mahmood A, Mahmood A, Malik RN, Shinwari ZK. Indigenous knowledge of medicinal plants from Gujranwala district, Pak J Ethnopharm 2013; 148:714-723. doi: https://dx.doi. org/10.1016/j.jep.2013.05.035

4. Gilani AH, Rahman A. Trends in ethnopharma. J Ethnopharm 2005; 100:43-49. doi: https://dx.doi. org/10.1016/j.jep.2005.06.001

5. Umair M, Altaf M, Abbasi AM. An ethnobotanical survey of indigenous medicinal plants in Hafizabad district, Punjab-Pakistan. PLoS One 2017; 12(6):e0177912. doi: https://dx.doi. org/10.1371/journal.pone.0177912

6. Alamgeer SA, Asif H, Younis W, Riaz H, Ishfaq $A B$, Assiri AM. Indigenous medicinal plants of Pakistan used to treat skin diseases: a review. Chinese Med 2018; 13:52-60. doi: https://dx.doi. org/10.1186/s13020-018-0210-0

7. Hussain K, Shahazad A, Zia-ul-Hussnain S. An ethnobotanical survey of important wild medicinal plants of Hattar district Haripur, Pakistan. Ethnobot Leaflet 2008; 12:29-35. https://opensiuc.lib.siu.edu/cgi/viewcontent. cgi?article $=1039 \&$ context $=\mathrm{ebl}$

8. Shinwari ZK. Ethnobotany in Pakistan: Sustainable and participatory approach. In Proc. 1st Training Workshop on Ethnobotany and its Application to Conservation NARC. J Biol Environ Sci 1996; 10(2):14-25. doi: https://dx.doi. org/10.1007/978-94-007-7747-7_9620

9. Khan AW, Khan A, Shah SMM, Ullah A, Faheem M, Saleem M. An updated list of neuromedicinal plants of Pakistan, their uses, and phytochemistry. Evidence-Based Complem Alter Med 2019; 1-28. doi: https://dx.doi. org/10.1155/2019/6191505

10. Shinwari ZK, Gilani SS. Sustainable harvest of medicinal plants at Bulashbar Nullah, Astore (northern Pakistan). J Ethnopharm 2003; 84(23):289-298. doi: https://dx.doi.org/10.1016/ S0378-8741(02)00333-1

11. Cunningham AB. Applied ethnobotany: people, wild plant use and conservation. Routledge 2014; 1:165-170.

12. Hussain K, Nisar MF, Majeed A. Ethnomedicinal survey for important plants of Jalalpur Jattan, district Gujrat, Punjab, Pakistan. Ethnobot Leaflet 2010; 7:11. https://opensiuc.lib.siu.edu/ebl/ vol2010/iss7/11/

13. Ali SS, Hussain K, Nawaz K, Bhatti KH, Bashir $\mathrm{Z}$, Nazeer A et al. Ethnobotanical knowledge and folk medicinal significance of the flora of district Gujrat, Punjab, Pakistan. Herba Polon 2020; 66(1):37-51. doi: https://dx.doi.org/10.2478/ hepo-2020-0004

14. Muhammad S, Khan Z, Cheema TA. Distribution of weeds in wheat, maize and potato fields of Tehsil Gojra, district Toba Tek Singh, Pakistan. Pak J Weed Sci Res 2009; 15(1): 91-105. https:// www.wssp.org.pk/weed/resources/images/ paper/955QW1450617779.pdf

15. Nasir E, Ali SI. Flora of Pakistan. No. 132-190. Islamabad, Karachi 1980.

16. Saurabh S, Camerota A, Zavotsky J. Gallstone ileus: diagnostic and therapeutic dilemma. Inter J Case Rep Images 2011; 2:1-5. doi: https://dx.doi. org/10.5348/ijcri-2011-12-71-CS-1 
17. Butt MA, Ahmad M, Fatima A. Ethnomedicinal uses of plants for the treatment of snake and scorpion bite in Northern Pakistan. J Ethnopharm 2015; 168:64-181. doi: https://dx.doi. org/10.1016/j.jep.2015.03.045

18. Akkol EK, Süntar I, Orhan IE. Assessment of dermal wound healing and in vitro antioxidant properties of Avena sativa L. J Cereal Sci 2011; 53: 285-90. doi: https://dx.doi.org/10.1016/j. jcs.2011.01.009

19. Kumar VL, Basu NO. Anti-inflammatory activity of the latex of Calotropis procera. J Ethnopharm 1994; 44(2):123-125. doi: https://dx.doi. org/10.1016/0378-8741(94)90078-7

20. Mishra G, Singh P, Verma R, Kumar S. Traditional uses, phytochemistry and pharmacological properties of Moringa oleifera plant: An overview. Der Pharmacia Lettre 2011; 3(2): 141-164. https:// www.scholarsresearchlibrary.com/articles/traditional-uses-phytochemistry-and-pharmacological-properties-of-moringa-oleifera-plant-anoverview.pdf

21. Padmavathi B, Rath PC, Rao AR. Roots of Withania somnifera inhibit for stomach and skin carcinogenesis in mice. Evidence-Based Compl Alter Med 2005; 2(1):99-105. doi: https://doi:10.1093/ ecam/neh064
22. Shinwari MI, Khan MA. Folk use of medicinal herbs of Margalla Hills National Park, Islamabad. J Ethnopharm 2000; 69(1):45-56. doi: https:// dx.doi.org/10.1016/S0378-8741(99)00135-X

23. Ahmad M, Qureshi R, Arshad M. Traditional herbal remedies used for the treatment of diabetes from district Attock (Pakistan). Pak J Bot 2009; 41(6):2777-2782. http://www.pakbs.org/ pjbot/PDFs/41(6)/PJB41(6)2777.pdf

24. Khan N, Abbasi AM, Dastagir G. Ethnobotanical and antimicrobial study of some selected medicinal plants used in Khyber Pakhtunkhwa (KPK) as a potential source to cure infectious diseases. BMC Comple Alter Med 2014; 14(1): 122-132. doi: https://dx.doi.org/10.1186/1472-6882-14-122

25. Qureshi R, Bhatti GR, Memon RA. Ethnomedicinal uses of herbs from northern part of Nara desert, Pakistan. Pak J Bot 2010; 42(2): 839851. http://www.pakbs.org/pjbot/PDFs/42(2)/ PJB42(2)0839.pdf 